\title{
Autogenous control augmentation system - A refinement in diced cartilage glue graft for augmentation of dorsum of nose
}

\author{
Gambhir Singh Swaroop, J. Sasidhar Reddy, Mahesh Chand Mangal, Anubhav Gupta, \\ Bheem Singh Nanda, Nikhil Jhunjhunwala \\ Department of Plastic and Cosmetic Surgery, Sir Ganga Ram Hospital, New Delhi, India
}

Address for correspondence: Dr. J. Sasidhar Reddy, Room No 2325, $3^{\text {rd }}$ Floor, SSRB Block, Old Rajinder Nagar, Sir Ganga Ram Hospital, New Delhi - 110 060, India. E-mail: j.sasidharreddy@gmail.com

\section{ABSTRACT}

Background: In the context of different grafts being used for dorsal augmentation, diced cartilage with glue has gained worldwide acceptance. Aims: To develop a system of tools to objectively evaluate the desired dimensions of the required graft for dorsal augmentation and to prepare a corresponding customized-glued-diced cartilage construct. Materials and Methods: A modification of the diced cartilage glue technique called Autogenous control augmentation system (ACAS) was used in ten patients. Results: Of the ten patients, in which this technique was used, eight underwent primary rhinoplasties and two underwent secondary rhinoplasties between July 2017 and December 2017 with a follow-up ranging from 3 to 8 months. In all the cases, the dorsum is straight, and height is maintained. Conclusion: The technique has all the advantages of the diced cartilage glue. The shape resembles alloplastic implant with height and width varying from radix to tip. There is tapering of the cephalic and caudal ends for more natural results. The brow tip aesthetic lines are better defined. The limitation of this study is short follow up.

\section{KEY WORDS}

Augmentation rhinoplasty; diced cartilage; fibrin glue

\section{INTRODUCTION}

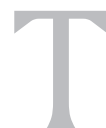

here is a constant endeavour to find an ideal material that can be used in the dorsal augmentation in rhinoplasty. The material should be of low cost, easily available, with low risk of infection and extrusion, and it should provide augmentation in a

\begin{tabular}{|l|l|}
\hline \multicolumn{2}{|c|}{ Access this article online } \\
\hline Quick Response Code: & Website: \\
\hline & www.ijps.org \\
\hline & Dol: \\
\hline
\end{tabular}

desired and more defined shape. Alloplastic implants, such as silicone and medpore, are being used, as they have a more defined shape, but have a risk of extrusion and infection. ${ }^{[1]}$ To combat this, there is a continuous search for autologous tissue that can fit all the requirements.

This is an open access journal, and articles are distributed under the terms of the Creative Commons Attribution-NonCommercial-ShareAlike 4.0 License, which allows others to remix, tweak, and build upon the work non-commercially, as long as appropriate credit is given and the new creations are licensed under the identical terms.

For reprints contact: reprints@medknow.com

How to cite this article: Swaroop GS, Reddy JS, Mangal MC, Gupta A, Nanda BS, Jhunjhunwala N. Autogenous control augmentation system - a refinement in diced cartilage glue graft for augmentation of dorsum of nose. Indian J Plast Surg 2018;51:202-7. 
The autologous tissues that are available are bone and cartilage. Bone graft has gone out of favour because of the high resorption rate, requirement of extra fixation and hard consistency. Cartilage can be used in solid form or diced form. The solid cartilage graft has less resorption than the bone; however, warping is the main problem. Different sources of solid cartilage grafts available are septum, concha and costal cartilage. The septum is a very common source of the graft. Volume available is adequate in primary rhinoplasties with no donor-site morbidity. The limitation is inadequate volume in graft-depleted patients and secondary rhinoplasties. With conchal graft, the donor scar is hidden, however, the graft is curved and not straight. ${ }^{[2]}$ Costal cartilage graft, usually taken from the $5^{\text {th }}, 6^{\text {th }}$ or $7^{\text {th }}$ ribs, is the largest source of cartilage. ${ }^{[3,4]}$ The disadvantages are donor-site pain and scar.

\section{How to preventing warping?}

There are a lot of techniques described to overcome warping; one of the techniques is counter-balancing technique $^{[5]}$ or "Namaste" technique. There is still a problem of graft show and firmness with this technique. Stacking the cartilage slices in a laminated fashion ${ }^{[6]}$ in alternate directions can reduce warping. Instead of suturing, people have tried to use cyanoacrylate in a porcine model, but found to have a strong foreign body reaction and resorption of grafts. Warping and resorption with cartilage crushing are uncontrolled. ${ }^{[7]}$ In allografting with irradiated rib, the donor-site problem and warping can be avoided, but the revision rate is high (26\%). ${ }^{[8,9]}$

\section{Diced cartilage with Surgicel ${ }^{\circledR} /$ fascia}

Erol, in the year 2000, published his technique of diced cartilage wrapped in Surgicel ${ }^{\circledR}$, calling it as "Turkish delight" and latter published his 26 years' experience in 2017. ${ }^{[10,11]}$ The long-term results were satisfactory with partial resorption in $0.4 \%$ and excessive resorption in $0.05 \%$. Daniel and Calvert tried to replicate the method $^{[12]}$ but found the higher resorptive rate which was attributed to the foreign body reaction seen from the histological study. In patients in whom Surgicel graft was used resorption of cartilage, was higher. So, they started using fascia ${ }^{[13]}$ covering over the diced cartilage and found that the chances of resorption was drastically reduced. They suggested a slight overcorrection as some resorption was still present. ${ }^{[14]}$ The disadvantages are an additional incision required in the temporal area and the extra time required to fill diced cartilage into the fascia bag. Different alternatives available to the fascia are hyaluronic acid membrane,
AlloDerm, Tutoplast-processed Allograft fascia lata, ${ }^{\mid 15]}$ rectus fascia and pectoralis major fascia. ${ }^{[16]}$

\section{Problems of fascia graft}

Regardless of whatever fascial graft used, the problem is that in the end, the graft becomes sausage shaped with a non-anatomical kidney-shaped cross section. There is no control of final height and width. Sometimes, if there is less filling of diced cartilage, a depression is noted in the dorsum. To circumvent these problems, various designer dorsal grafts came into use such as combination of bare-diced cartilage and diced cartilage under the fascia. Still, it is very difficult to make anatomically correct crescent-shaped (cross section) graft with tapering sides.

\section{Fibrin glue}

Tasman has introduced fibrin glue to fix diced cartilage together as a single unit. ${ }^{[17,18]} \mathrm{He}$ moulded it over a longitudinally cut 2 or $5 \mathrm{ml}$ syringe. Glue stands as a good alternative to the fascia. There are reports of usage of autologous tissue glue such as PRP (platelet gel), PPP (fibrin glue) and whole blood. ${ }^{[19]}$

We, at our institute, have designed a technique to objectively evaluate the desired dimensions of the required graft and to prepare a customized-diced cartilage graft.

\section{MATERIALS AND METHODS}

A modification of the diced cartilage glue technique called Autogenous Control Augmentation System (ACAS) was used in ten patients.

\section{Technique}

Open rhinoplasty approach was used and the augmentation of the dorsum was the last step of the operative procedure. For this, we used the Tasman technique with few modifications of our own [Figures 1-6]. The harvested cartilage is diced into $0.2-0.3 \mathrm{~mm}$ pieces. They are then transplanted into the $1 \mathrm{ml}$ syringe. The extra saline was then taken out by pressing the syringe into the gauze. The concentrated diced cartilage was ready in the syringe for the use. We use reusable sizers made of silicone (normally available as implants). They are of various sizes and shapes. We place them into the pocket in the nose. We thus select a sizer of the desired height, length and width and sculpt it if necessary.

We have made different sizes of moulds in steel plate just like the sizers. We call it autogenous control augmentation 


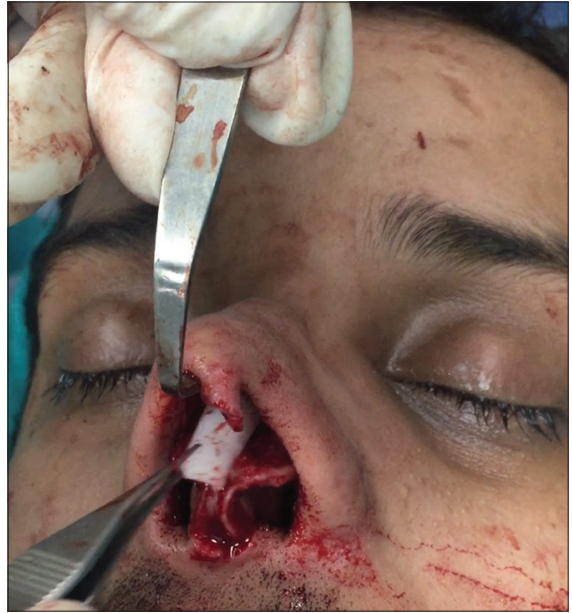

Figure 1: Placement of sizer in the pocket over the dorsum of the nose

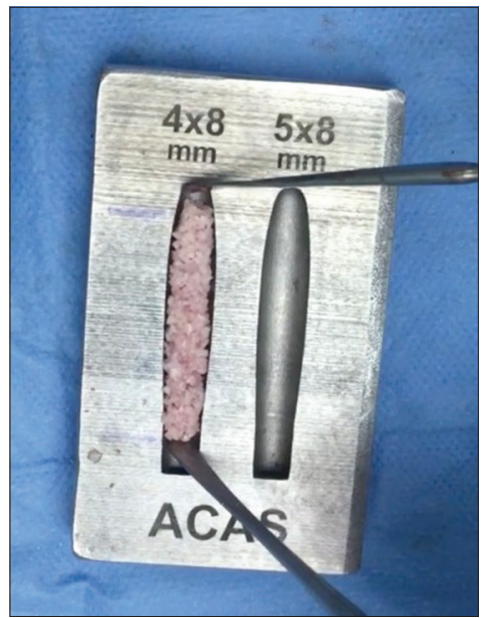

Figure 3: Placing diced cartilage in the mould

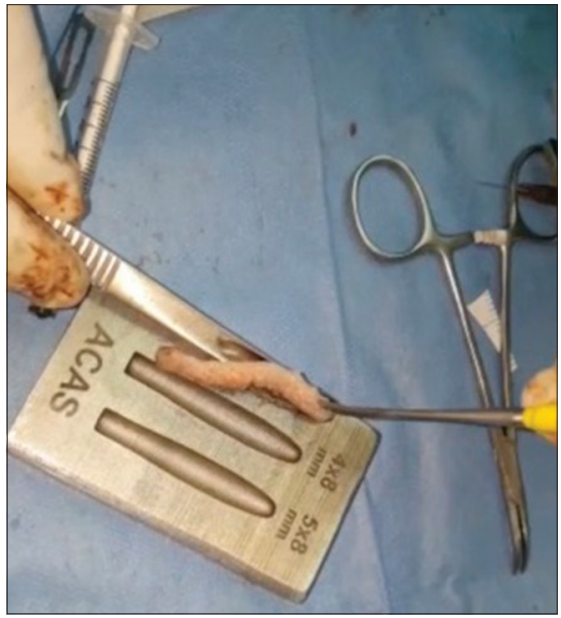

Figure 5: Placing the glued-diced cartilage graft onto a carrier

system [Figure 2]. The diced cartilage from the syringe is then put into the mould corresponding to the sizer used [Figure 3]. First, thrombin is put and mixed with freer elevator then we put fibrin and let it polymerise Indian Journal of Plastic Surgery Volume 51 Issue 2 May-August 2018

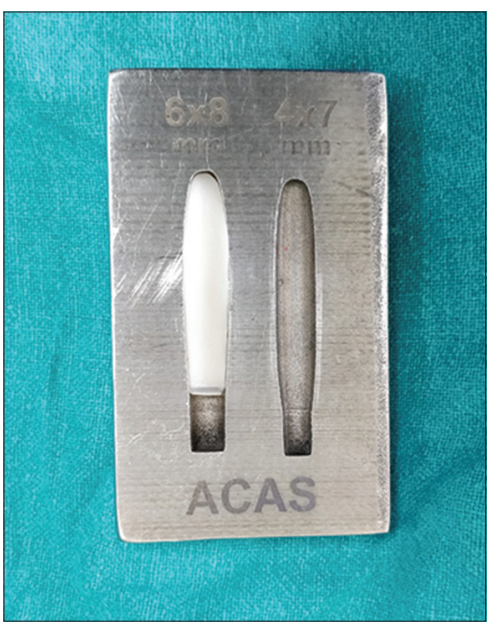

Figure 2: Finding the appropriate mould for the sizer to fit in

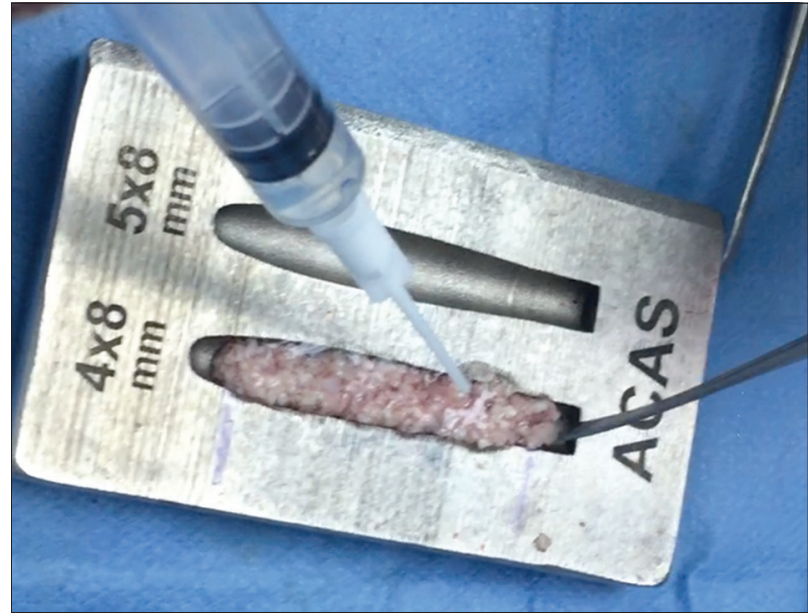

Figure 4: Placing fibrin glue in the diced cartilage

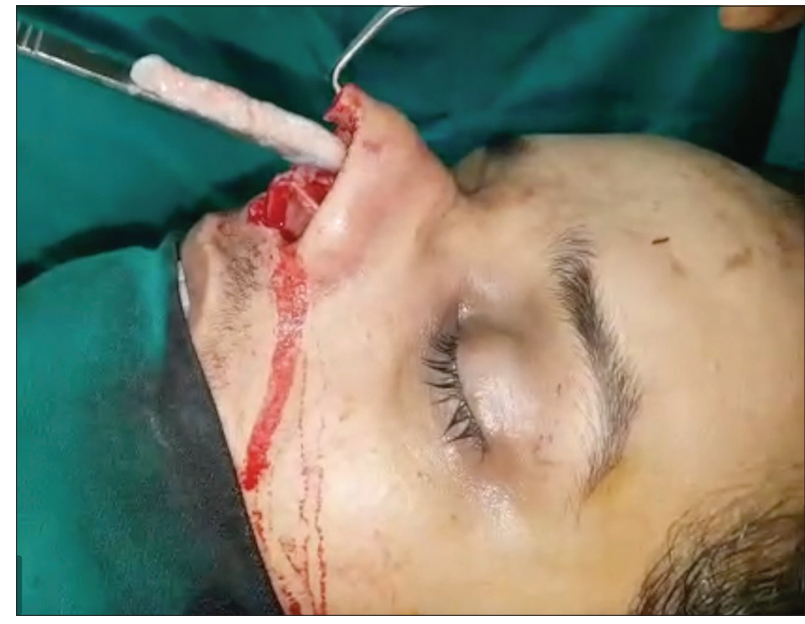

Figure 6: Introducing the graft in the pocket of the nasal dorsum

for $20-30 \mathrm{~s}$. The mould is ready in $3-5$ min [Figure 4]. A pocket over the dorsum has to be dissected prior. We gently lift the mould into the carrier [Figure 5] and then place it into the dorsum carefully by retracting the skin 
flap with an Aufricht retractor. The radix point or the planned cephalic end of the mould is marked earlier. The caudal end of the mould reaches the supratip area.

All the three dimensions length, width and height of each mould are fixed and different. We can even prepare a glued cartilage graft of less length and height of each corresponding mould.

\section{RESULTS}

Of the ten patients, in which this technique was used, eight underwent primary rhinoplasties and two underwent secondary rhinoplasties between July 2017 and December 2017 with a follow-up ranging from 3 to 8 months. In all the cases, the dorsum is straight and height is maintained [Figures 7-9]. In one case, slight deviation was observed at the cephalic end of the graft in the $3^{\text {rd }}$ week, which got corrected by gentle massage. The average dimensions of the grafts used were $33.2 \mathrm{~mm}$ (length), $8.7 \mathrm{~mm}$ (maximum width) and $4.5 \mathrm{~mm}$ (maximum height) [Table 1].

\section{DISCUSSION}

Ideal tissue or material for dorsal augmentation is yet to be found, but diced cartilage has gained worldwide acceptance for its ability to survive with or without fascia graft.

The disadvantages with using a fascia graft are another donor site added and the extra time consumed in

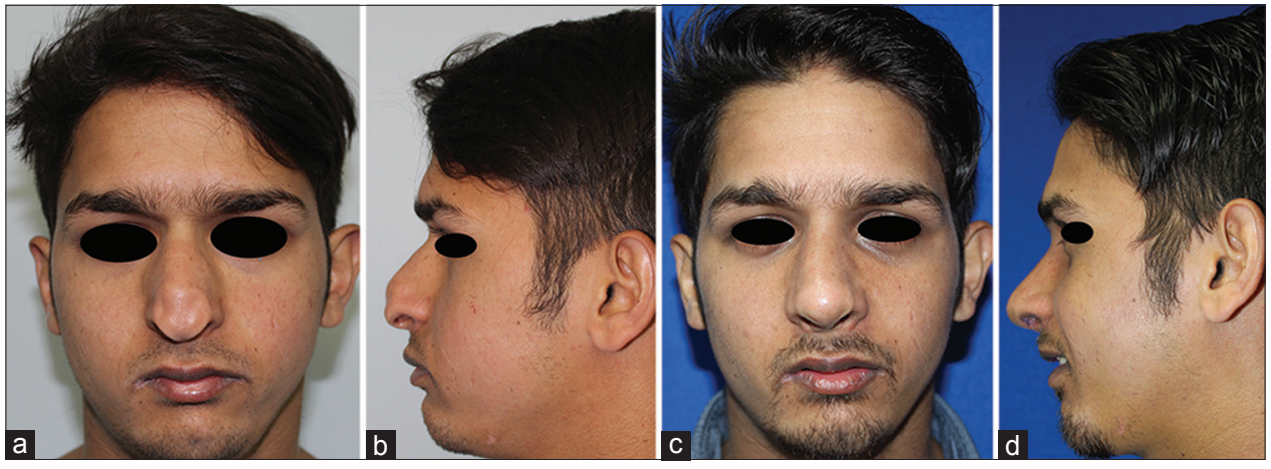

Figure 7: (a) Patient 1-pre-operative. (b) Patient 1-pre-operative. (c) Patient 1-post-operative. (d) Patient 1-post-operative
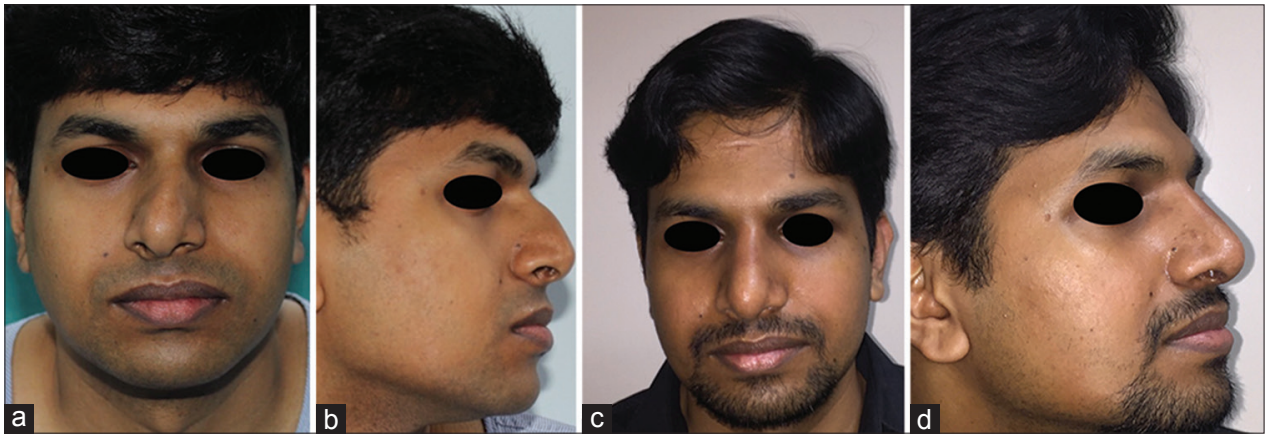

Figure 8: (a) Patient 2-pre-operative. (b) Patient 2-pre-operative. (c) Patient 2-post-operative. (d) Patient 2-post-operative
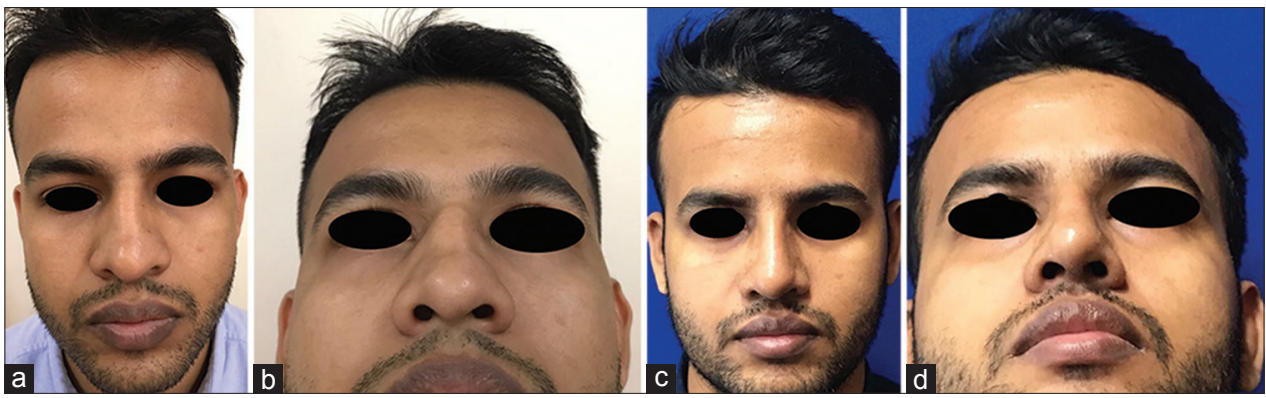

Figure 9: (a) Patient 3-pre-operative. (b) Patient 3-pre-operative. (c) Patient 3-post-operative. (d) Patient 3-post-operative 
Table 1: Dimensions of the grafts used

\begin{tabular}{lccc}
\hline Case & Length $(\mathbf{m m})$ & Width $(\mathbf{m m})$ & Height $(\mathbf{m m})$ \\
\hline 1 & 35 & 7 & 3 \\
2 & 29 & 8 & 4 \\
3 & 37 & 10 & 3 \\
4 & 30 & 8 & 4 \\
5 & 33 & 8 & 6 \\
6 & 32 & 8 & 5 \\
7 & 35 & 10 & 6 \\
8 & 34 & 10 & 5 \\
9 & 33 & 8 & 4 \\
10 & 34 & 10 & 5 \\
\hline
\end{tabular}

preparing the sleeve and putting diced cartilage into it. Using Tutoplast (Alloplastic fascia lata), we can solve these problems but still end up having a sausage-shaped bag. ${ }^{[20]}$ This shape is not anatomical, and there may be depression in long term if diced cartilage is not filled tightly. They are not very predictable.

The ideal shape to attain is a crescent-shaped cross section, which is difficult to achieve with diced cartilage fascia and solid rib graft.

It is already proven that fibrin glue (tisseel) used to form the scaffold to bind the cartilage is replaced by fibrous tissue in the long term. The fibrin glue causes chondrocyte proliferation and cartilaginous tissue formation with the development of cartilage-specific extracellular matrix components glycosaminoglycans and collagen Type II. ${ }^{[1]}$

The main drawback of the Tasman technique using fibrin glue in a syringe to form the scaffold is that there is very difficult to assess the height and width of the graft. The graft is uniform throughout and gives a parallel line equidistant from tip to radix. There is no good control of the parameters.

As of now, there is no system of tools to objectively evaluate the desired dimensions of the required graft and to prepare a corresponding customized-glued-diced cartilage construct. The technique which we have developed has all the advantages of the diced cartilage glue. As well, it is more silastic shaped, with height and width varying from radix to tip. There is tapering of the cephalic and caudal ends for more natural results. The brow tip aesthetic lines are better defined. It can be moulded by manual pressure in the $1^{\text {st }}$ post-operative weeks, and there is no dead space present as in fascia graft. The main advantage of this technique is that it is more objective and controlled to give better results.
Though the initial clinical outcome is promising the long term results remains to be seen.

\section{CONCLUSION}

The technique has all the advantages of the diced cartilage glue. As well, it is more alloplastic implant shaped with height and width varying from radix to tip. There is tapering of the cephalic and caudal ends for more natural results. The brow tip aesthetic lines are better defined. The limitation of our study is short follow up.

\section{Declaration of patient consent}

The authors certify that they have obtained all appropriate patient consent forms. In the form the patient(s) has/have given his/her/their consent for his/her/their images and other clinical information to be reported in the journal. The patients understand that their names and initials will not be published and due efforts will be made to conceal their identity, but anonymity cannot be guaranteed.

\section{Financial support and sponsorship}

Nil.

\section{Conflicts of interest}

There are no conflicts of interest.

\section{REFERENCES}

1. Sajjadian A, Rubinstein R, Naghshineh N. Current status of grafts and implants in rhinoplasty: Part I. Autologous grafts. Plast Reconstr Surg 2010;125:40e-9e.

2. Kim JH, Jang YJ. Use of diced conchal cartilage with perichondrial attachment in rhinoplasty. Plast Reconstr Surg 2015;135:1545-53.

3. Lee M, Inman J, Ducic Y. Central segment harvest of costal cartilage in rhinoplasty. Laryngoscope 2011;121:2155-8.

4. Chauhan N, Sepehr A, Gantous A. Costal cartilage autograft harvest: Inferior strip preservation technique. Plast Reconstr Surg 2010;125:214e-5e.

5. Agrawal KS, Bachhav M, Shrotriya R. Namaste (counterbalancing) technique: Overcoming warping in costal cartilage. Indian J Plast Surg 2015;48:123-8.

6. Swanepoel PF, Fysh R. Laminated dorsal beam graft to eliminate postoperative twisting complications. Arch Facial Plast Surg 2007;9:285-9.

7. Cakmak O, Buyuklu F. Crushed cartilage grafts for concealing irregularities in rhinoplasty. Arch Facial Plast Surg 2007;9:352-7.

8. Clark RP. Nasal dorsal augmentation with freeze-dried allograft bone. Plast Reconstr Surg 2010;126:1113-6.

9. Kridel RW. Ashoori long-term use with follow up afirrashated homologous costal cartilage graft in $\mathrm{Vn}$ nose. Arch Fac Plast Surg 2009;11:378-94.

10. Erol OO. The Turkish delight: A pliable graft for rhinoplasty. Plast Reconstr Surg 2000;105:2229-41.

11. Erol OO. Long-term results and refinement of the Turkish delight 
technique for primary and secondary rhinoplasty: 25 years of experience. Plast Reconstr Surg 2016;137:423-37.

12. Calvert JW, Brenner K, DaCosta-lyer M, Evans GR, Daniel RK. Histological analysis of human diced cartilage grafts. Plast Reconstr Surg 2006;118:230-6.

13. Daniel RK, Calvert JW. Diced cartilage grafts in rhinoplasty surgery. Plast Reconstr Surg 2004;113:2156-71.

14. Kim EK, Daniel RK. Operative techniques in Asian rhinoplasty. Aesthet Surg J 2012;32:1018-30.

15. Jang YJ, Song HM, Yoon YJ, Sykes JM. Combined use of crushed cartilage and processed fascia lata for dorsal augmentation in rhinoplasty for Asians. Laryngoscope 2009;119:1088-92.

16. Cerkes N, Basaran K. Diced cartilage grafts wrapped in rectus abdominis fascia for nasal dorsum augmentation. Plast Reconstr Surg 2016;137:43-51.
17. Tasman AJ, Diener PA, Litschel R. The diced cartilage glue graft for nasal augmentation. Morphometric evidence of longevity. JAMA Facial Plast Surg 2013;15:86-94.

18. Tasman AJ. Advances in nasal dorsal augmentation with diced cartilage. Curr Opin Otolaryngol Head Neck Surg 2013;21:365-71.

19. Codazzi D, Ortelli L, Robotti E. Diced cartilage combined with warm blood glue for nasal dorsum enhancement. Aesthetic Plast Surg 2014;38:822-3.

20. Gordon CR, Alghoul M, Goldberg JS, Habal MB, Papay F. Diced cartilage grafts wrapped in alloDerm for dorsal nasal augmentation. J Craniofac Surg 2011;22:1196-9.

21. Özkan A, Topkara A, Akbulut M, Özcan RH. Survival of minced cartilage grafts with comparison surgicel( $($ ) original and fibrillar. Aesthetic Plast Surg 2016;40:602-12. 